\title{
SHARP - II. Mass structure in strong lenses is not necessarily dark matter substructure: A flux ratio anomaly from an edge-on disc
}

\author{
J.-W. Hsueh, ${ }^{1 \star}$ C. D. Fassnacht, ${ }^{1}$ S. Vegetti, ${ }^{2}$ J. P. McKean,,${ }^{3,4}$ C. Spingola, ${ }^{4}$ M. W. Auger ${ }^{5}$ \\ L. V. E. Koopmans, ${ }^{4}$ and D. J. Lagattuta ${ }^{6}$ \\ ${ }^{1}$ Department of Physics, University of California, Davis, 1 Shields Ave. Davis, CA 95616, USA \\ ${ }^{2}$ Max Planck Institute for Astrophysics, Karl-Schwarzschild-Strasse 1, D-85740 Garching, Germany \\ ${ }^{3}$ Netherlands Institute for Radio Astronomy (ASTRON), P.O. Box 2, 7990 AA Dwingeloo, The Netherlands \\ ${ }^{4}$ Kapteyn Astronomical Institute, University of Groningen, P.O. Box 800, 9700 AV Groningen, The Netherlands \\ ${ }^{5}$ Institute of Astronomy, University of Cambridge, Madingley Road, Cambridge CB3 OHA, UK \\ ${ }^{6}$ University of Lyon, CRAL, Observatoire de Lyon, 92 Rue Pasteur, 69007 Lyon, France
}

15 October 2018

\begin{abstract}
Gravitational lens flux-ratio anomalies provide a powerful technique for measuring dark matter substructure in distant galaxies. However, before using these flux-ratio anomalies to test galaxy formation models, it is imperative to ascertain that the given anomalies are indeed due to the presence of dark matter substructure and not due to some other component of the lensing galaxy halo or to propagation effects. Here we present the case of CLASS B1555+375, which has a strong radio-wavelength flux-ratio anomaly. Our high-resolution near-infrared Keck II adaptive optics imaging and archival Hubble Space Telescope data reveal the lensing galaxy in this system to have a clear edge-on disc component that crosses directly over the pair of images that exhibit the flux-ratio anomaly. We find that simple models that include the disc can reproduce the $\mathrm{cm}$-wavelength flux-ratio anomaly without requiring additional dark matter substructure. Although further studies are required, our results suggest the assumption that all flux-ratio anomalies are due to a population of dark matter sub-haloes may be incorrect, and analyses that do not account for the full complexity of the lens macro-model may overestimate the substructure mass fraction in massive lensing galaxies.
\end{abstract}

Key words: gravitational lensing - quasars: individual CLASS B1555+375 - galaxies: structure

\section{INTRODUCTION}

A common feature of dark-matter only simulations of structure formation is the presence of thousands of subhaloes that are associated with larger mass haloes (e.g. Springel et al. 2008). However, observations of the Local Group show many fewer satellite galaxies than are predicted by the simulations, even when taking into account the latest discoveries of new dwarf galaxies (The DES Collaboration et al. 2015; Koposov et al. 2015) and completeness corrections arising from the limited sky coverage imposed by the Galactic Plane. This is the well-known missing satellite problem (Klypin et al. 1999; Moore et al. 1999; Strigari et al. 2007). In order to understand this discrepancy, it is imperative to build up a large sample of satellites/subhaloes in galaxies outside the Local Group. However, this process is challenging due to the faintness of the satellite galaxies. In fact, lower-mass satellites may not be able to retain the gas needed for ongoing star formation (e.g. Papastergis et al. 2011), rendering them effectively dark.

^ E-mail: jwhsueh@ucdavis.edu
It was first suggested by Mao \& Schneider (1998) that the flux-ratio anomalies observed in the multiple radio-loud images of lensed quasars could be interpreted as a sign of the presence of substructure in lens galaxies. Indeed, small perturbations in the gravitational potential are sufficient to cause flux-ratio anomalies in gravitationally lensed systems (Metcalf \& Madau 2001; Dalal \& Kochanek 2002; Bradač et al. 2002), particularly for those systems with a close pair of merging images (Kochanek \& Dalal 2004). At radio wavelengths, the lensed images are neither sensitive to micro-lensing from stars (Koopmans et al. 2003; although see Koopmans \& de Bruyn 2000), nor to dust extinction. Also, any variation in the properties of the radio images that are due to propagation effects (e.g. interstellar scattering or free-free absorption) have a well understood frequency dependence that can be identified and accounted for (e.g. Biggs et al. 2003; Mittal et al. 2007; Winn et al. 2004). This makes the flux-ratio anomalies of radio-loud lensed images a promising tool to detect and measure dark matter substructures in a large sample of distant galaxies (Dalal \& Kochanek 2002). However, a recent comparison of the observational data (eight gravitational lens systems with four or 



Figure 1. High-resolution multi-band imaging of B1555+375 with HST/WFPC2 at F555W (left) and F814W (middle-left), with HST/NICMOS/NIC2 at F160W (middle-right) and with Keck adaptive optics at $K^{\prime}$-band (right). All of the panels are 3.7 arcsec on a side and the scale-bar represents 1 arcsec.

more images) with the predictions from a set of cold dark matter simulations that are representative of the mass and redshift of the lens galaxies suggests that there is only a $1-4$ per cent probability that the full observed flux-ratio distribution is produced only by substructure (Xu et al. 2015).

The gravitational imaging technique, which alternatively utilizes the surface brightness distribution of extended arcs and Einstein rings to detect low-mass substructures within lensing haloes, was introduced by Koopmans (2005) and Vegetti \& Koopmans (2009). This method has given a measurement of the substructure mass fraction for a sample of 11 lenses that is currently in agreement with the theoretical expectations from cold matter matter simulations (Vegetti et al. 2014, 2012). The reason for the conflicting results between the flux-ratio anomaly and gravitational imaging techniques is currently not clear. While this could be the result of small sample statistics, it could also be an indication that the fluxratio anomalies have a different origin than clumpy substructure (see Xu et al. 2015, for a discussion).

In this letter, we explore the idea that not all flux-ratio anomalies are due to the presence of clumpy substructure, but that some originate from more complex lens galaxy mass distributions than have initially been considered. To this end, we use new infrared and radio interferometric imaging data from the Strong lensing at High Angular Resolution Programme (SHARP; McKean et al. 2007; Lagattuta et al. 2010, 2012; Vegetti et al. 2012, Chen et al. 2016 submitted). The main goal of SHARP is to investigate galaxy structure and other astrophysical topics through the re-observation of known gravitational lens systems at high angular resolution, making use of observations with (1) adaptive optics (AO) on the W. M. Keck telescope, (2) the Hubble Space Telescope (HST), and (3) large radio and $\mathrm{mm}$ interferometric arrays.

Here we focus on CLASS B1555+375 (Marlow et al. 1999), which was discovered as part of the Cosmic Lens All-Sky Survey (Myers et al. 2003; Browne et al. 2003). B1555+375 has four lensed images with a maximum separation of 426 mas in a fold configuration. The two merging lensed images show a strong and persistent flux-ratio anomaly at radio wavelengths, and previous radio monitoring observations ruled out microlensing effects as the cause of the anomaly (Koopmans et al. 2003). As B1555+375 was included in the observational and theoretical analyses of both Dalal \& Kochanek (2002) and Xu et al. (2015), understanding its mass distribution is of key importance to assess any systematics with the inferred level of substructure in the lensing halo with the flux-ratio anomaly method. The SHARP imaging data are introduced in Section 2 and we present a revised lens model using these data in Section 3. The results of this analysis and their implications are discussed in Section 4. Note that the redshifts of both the lens galaxy and the lensed source are unknown for B1555+375, so we assume redshifts of $z_{l}=1$ and $z_{s}=1.5$, respectively, due to their red colours (Marlow et al. 1999). This assumption has no effect on the derived magnifications or flux ratios of our analysis.

\section{OBSERVATIONS \& DATA REDUCTION}

Our dataset consists of optical and infrared imaging taken with the W. M. Keck-II Telescope and HST, and high-resolution radio imaging taken with the Very Long Baseline Array (VLBA), which are discussed below and are summarized in Table 1.

\subsection{Keck adaptive optics and $H S T$ imaging}

B1555+375 was observed as part of SHARP using the NIRC2 camera on the W. M. Keck-II Telescope on 2012 May 16 (PI: Fassnacht). The AO system was used, with the corrections derived from the laser guide star and a $R=14$.4-mag tip-tilt star located $\sim 45$ arcsec from the lens. The narrow camera mode was used, giving a field of view of $\sim 10 \operatorname{arcsec}$ on a side and a pixel scale of 10 mas. Six dithered $300 \mathrm{~s}$ exposures were obtained in the $K^{\prime}$ filter. The data were reduced with the standard SHARP pipeline, which is a python-based package that is a refinement of the process described by Auger et al. (2011). A cutout of the final reduced image is shown in Fig. 1 and also in Fig. 2a, where the contours from 5-GHz MultiElement Radio Link Interferometer Network (MERLIN) imaging by Marlow et al. (1999) and 1.66-GHz VLBA imaging (see below) are overlaid.

B1555+375 was also observed with the HST in three broad bands. The optical data were obtained with the Wide-Field Planetary Camera 2 (WFPC2) in the F555W and F814W bands (GO8804; PI: Falco), while the Near Infrared Multi-Object Spectrograph (NICMOS) was used to observe the system in the F160W band (GO-9744; PI: Kochanek). The NICMOS observations were obtained with the NIC2 camera. We reduced all of the archival HST data with the standard MULTIDRIzZLE pipeline, producing final drizzled images with pixel-sizes of 50 mas. The reduced images are also shown in Fig. 1. The lens and the lensed images are not detected at high significance in the optical bands, but are clearly seen in the NICMOS F160W image.

There are several notable features in the high-resolution AO and HST data. These include the nearly complete lack of emission associated with lensed images B and D, (Fig. 2b), and the faint, but clearly visible emission from the lensing galaxy that appears to be 
Table 1. Summary of the B1555+375 observations.

\begin{tabular}{llccc}
\hline Telescope & Camera & Band & Date & $t_{\text {exp }}(\mathrm{s})$ \\
\hline VLBA & & $1.66 \mathrm{GHz}$ & 2000 Mar 21 & 10800 \\
$H S T$ & WFPC2 & F555W & 2000 Oct 09 & 5200 \\
$H S T$ & WFPC2 & F814W & 2000 Oct 09 & 5200 \\
$H S T$ & NICMOS/NIC2 & F160W & 2003 Nov 02 & 5376 \\
Keck-II & NIRC2 AO & $K^{\prime}$ & 2012 May 16 & 1800 \\
\hline
\end{tabular}

an edge-on disc, with a position angle of $\sim 10$ deg and an axis ratio $q \sim 0.2$. We note that the position angle of the lensing galaxy is such that the disc emission lies close to the lensed images B and D. Therefore, the lack of emission from these rest-frame optical images is likely due to dust extinction and additional demagnification due to lensing.

\subsection{Very Long Baseline Array imaging}

B1555+375 was observed with the 10 telescopes of the VLBA at a central observing frequency of $1.66 \mathrm{GHz}$ on 2000 March 21 (BN0009; PI: Norbury). The data were recorded at $128 \mathrm{Mbits} \mathrm{s}^{-1}$ and then correlated to produce two spectral windows with $8 \mathrm{MHz}$ bandwidth, 16 channels and both circular polarizations (RR and LL). The observations were phase referenced using J1544+398 every $3 \mathrm{~min}$ over the total observing time of $3 \mathrm{~h}$. The data were reduced in the standard manner using the vLBAUTLIs analysis pipeline that is part of the Astronomical Image Processing Software (AIPS). Imaging was done with the CLEAN algorithm in AIPS using natural weighting of the visibilities, and restored using an elliptical beam of size $9.7 \times 6.9$ mas at a position angle of $-7.6^{\circ}$ east of north. The final map (also shown in Fig. 2a) has an rms of $78 \mu \mathrm{Jy} \mathrm{beam}^{-1}$.

We find that lensed images $\mathrm{A}$ and $\mathrm{B}$ have been resolved into a gravitational arc that is $\sim 100$ mas in length. Image $\mathrm{C}$ is still compact on mas-scales, whereas image $\mathrm{D}$ is only marginally detected $(\sim 6 \sigma)$. The VLBA imaging shows that image B has a smaller angular size than image $\mathrm{A}$, which is consistent with a perturbation in the mass model being responsible for the flux-ratio anomaly (recall that gravitational lensing conserves surface brightness), as opposed to free-free absorption or interstellar scattering.

\section{LENS MODELLING}

The flux-ratio anomaly and relative sizes of the images at radiowavelengths imply that there must be some form of perturbation to the gravitational lens mass model, which has thus far been attributed to dark matter substructure within the lensing galaxy or along the line of sight to the lensed images (Dalal \& Kochanek 2002; Xu et al. 2012, 2015). However, our high-resolution near infrared imaging suggests the intriguing possibility that the mass perturbation could be due to an edge-on disc component within the lens. To test this possibility, we model the system with a disc component in order to see if a plausible mass model can explain the flux-ratio anomaly without the need for additional substructure.

We use the lens modelling code GRAVLENs (Keeton 2001) to model the compact radio components. The inputs to the model are the observed image positions measured from the MERLIN radio observations of Marlow et al. (1999) as all of the images are significantly detected in these data. The flux-ratio measurements are taken from Koopmans et al. (2003), who used half a year of MERLIN monitoring to obtain flux-ratio curves. In total, there are 11 constraints to the mass model provided by the observational data.
As a first trial of modelling, we re-create the singular isothermal ellipsoid without external shear model (SIE; 7 free parameters) of Marlow et al. (1999) to check the performance of a simple lensing potential. Consistent with previous studies (Marlow et al. 1999; Xu et al. 2015), a single elliptical potential model cannot reproduce the observed flux ratios in B1555+375 (see Table 2).

The next step is to test if a physically plausible edge-on disc can cause a perturbation in the strong lensing mass model that produces the observed flux ratio anomaly of merging images $\mathrm{A}$ and B. An exponential disc profile best describes the disc component in spiral galaxies because it matches the light distribution (Keeton \& Kochanek 1998). We thus choose a SIE plus an exponential disc model (SIE+ExpDisc) in our study of B1555+375. The free parameters of this model are the SIE Einstein radius $(b)$, centroid position, ellipticity $(e=1-q)$, position angle $(\theta)$, the exponential disc intrinsic central density $\left(\kappa_{0}\right)$ and scale length $\left(R_{S}\right)$. A detailed definition of these mass profiles is described by Keeton (2001). The source position adds two additional model parameters. We have not included a third component such as an external shear or additional NFW halo term because the number of constraints provided by the compact images detected by MERLIN is not sufficient to constrain such a model.

More sophisticated mass models require additional constraints, such as those from the lensed extended emission in either the NIR or radio imaging. While we have successfully used the former approach in the past (e.g. Vegetti et al. 2012), for B1555+375 the extinction in the $\mathrm{AO}$ imaging is so severe that components $\mathrm{B}$ and $\mathrm{D}$ are not visible, and the arc that connects $\mathrm{A}$ and $\mathrm{B}$ appears to be truncated. The low signal-to-noise ratio in the ring away from the lensed AGN and the strong extinction in the lens galaxy prevent us from using this gravitational imaging approach. Significantly deeper observations are needed at a higher bandwidth with very long baseline interferometric arrays, which can test more complex models in the future by imaging the extended gravitational arc and more significantly detecting image $\mathrm{D}$.

We use GRAVLENS to obtain the best-fit SIE+ExpDisc lens model. The best-fit parameters from Marlow et al. (1999) are chosen as the starting point of the SIE profile. We have used the NICMOS F160W imaging, in which the disc component has the highest signal-to-noise ratio amongst our data, to fix the disc centroid position relative to image A to $(-0.162,-0.206)$, its ellipticity to $e=0.83$, and its position angle to $\theta=8^{\circ}$. The total number of free parameters is 9 . After obtaining the best-fit lens model, we run a Markov-Chain Monte-Carlo (MCMC) analysis to obtain the uncertainties.

The best-fit model parameters and 68 per cent confidence level (CL) errors are listed in Table 3 and the lens model is illustrated in Fig. 2c. Table 2 also shows the comparison between the observed radio data and the model-predicted image positions and flux ratios. We find that by adding an elongated exponential disc to a smooth SIE model there is significant improvement in the predicted flux ratios when compared to the results for the SIE-only model. The positions are all in agreement, and the flux ratios are consistent at the $2 \sigma$ level, with excellent agreement now obtained for the two merging images $\mathrm{A}$ and $\mathrm{B}$. The remaining differences in the fluxes may be due to the real lensed source being slightly extended rather than the point source that we used in our model. The model requires that the ExpDisc component makes up about 15 per cent of the total projected mass within the Einstein radius (1.7 kpc for an assumed lens redshift of $z_{l}=1$ but the mass fraction is independent of the lens and source redshifts since this ratio is independent of distance). In this central region of an edge-on spiral galaxy, the baryonic mass 
can be a significant component of the total projected mass. Investigations of edge-on spiral lenses, for example from the SWELLS survey, show that the disc mass fraction within the Einstein radius ranges from 5 to 30 per cent(Treu et al. 2011; Brewer et al. 2012, 2014).

\section{DISCUSSION \& CONCLUSIONS}

Although flux-ratio anomalies provide a powerful tool to investigate low-mass substructure in lensing haloes out to cosmological redshifts, it is clear that some care is needed in interpreting the results. It is now well established that observations of radioloud gravitational lenses may provide the most robust observational constraints because they are less affected by dust extinction and microlensing, and monitoring can determine the best estimate of the intrinsic flux ratios. This has led to an oft-studied sample of eight radio-loud gravitational lenses with well-defined flux-ratio measurements (Dalal \& Kochanek 2002; Kochanek \& Dalal 2004; $\mathrm{Xu}$ et al. 2015). In general, lens models that are based only on the locations of lensed radio-loud images have large degeneracies due to the limited number of constraints that are provided by 50 to 200 mas imaging of the unresolved sources (e.g. Kochanek 1991). Furthermore, observed flux-ratio anomalies may not be due exclusively to a population of low-mass "dark" substructures, as already seen in the MG J0414+0534 and MG J2016+112 systems, where there is evidence for luminous companion dwarf galaxies that can account for the observed flux ratios, and even observed astrometric anomalies (Ros et al. 2000; Chen et al. 2007; More et al. 2009; see also McKean et al. 2007; Jackson et al. 2010).

Here, we find evidence for an edge-on disc in the flux-ratio anomaly gravitational lens B1555+375 that can account for the required perturbation in the mass model. Although a constraint on the disc mass from photometry is currently not available due to the low signal-to-noise ratio in the $\mathrm{AO}$ imaging and the lack of redshift information, our model indicates that the flux-ratio anomaly in B1555+375 has a strong link to the presence of an edge-on disc. Such high-order deviations from a smooth mass distribution have already been suggested previously (Evans \& Witt 2003; Congdon \& Keeton 2005), but this is the first time that an observed disc has been explicitly modelled to account for a fluxratio anomaly. However, it is important to realise that by including observationally-motivated modifications to a 'smooth' mass distribution, such as luminous dwarf companions or edge-on discs, in the mass models, we are still limited in our interpretation if we can not determine how much of the anomaly are due to these components. For our current analysis of B1555+375, we have shown that the entire anomaly could be caused by the edge-on disc. That is not to imply this is a unique model, but rather that the disc component is a plausible explanation of the anomaly.

In future it may be possible to independently constrain the mass of the disc through kinematic measurements (most likely from mm-wavelength resolved emission line observations), which would test whether the disc actually makes a sufficient contribution to the mass model (e.g. as in the case of B1933+503; Suyu et al. 2012). Also, in the blue contours of Fig. 2a, B1555+375 shows evidence for an extended $\sim 100$ mas gravitational arc at radio wavelengths that crosses the edge-on disc. Further high resolution imaging with very long baseline interferometry will provide the observational constraints required to fully test whether the disc is sufficient to cause the anomaly.

Our analysis of B1555+375 demonstrates that the choice of the macromodel used to describe the 'smooth' lens galaxy mass distribution, combined with the limited information provided by the position of compact lensed images, can lead to a misinterpretation of the origin of flux-ratio anomalies in some systems. Special care may be needed for small-separation lens systems, in which the lensing galaxy is likely to be a spiral (e.g., Turner et al. 1984; Fukugita et al. 1992). This is because a disc with a high inclination angle can significantly affect the lensing cross-section for such systems (Maller et al. 1997; Keeton \& Kochanek 1998; Möller et al. 2003). The current sample of lensed quasars with radio-wavelength flux ratio measurements is small. Therefore, having even a few systems with edge-on discs may be sufficient to introduce a bias into the substructure analysis. In fact, our near-infrared $\mathrm{AO}$ imaging of other lenses in the SHARP sample shows that B1555+375 is not the only system that has a significant disc component that crosses the merging lensed images (Fassnacht et al., in prep.). These gravitational lenses will be investigated in future work, but the images alone suggest that other systems may have a similar link between flux-ratio anomalies and disc components. This potential bias introduced by edge-on discs may be avoided by post-selection as sample sizes grow in the future. Therefore, it is critical to obtain high-resolution imaging lenses to detect additional mass features such as edge-on discs or even massive luminous satellites. This result is timely, as upcoming large-scale surveys (e.g., the Dark Energy Survey, Kilo-Degree Survey, Euclid, and the Large Synoptic Survey Telescope) will deliver orders of magnitude more gravitationally lensed quasars for which flux ratios can be measured using narrow lines (e.g. Nierenberg et al. 2014).

\section{ACKNOWLEDGMENTS}

We thank the referee for comments that improved the paper. We are grateful to Dandan $\mathrm{Xu}$ for useful discussions. CDF and DJL acknowledge support from NSF-AST-0909119. LVEK is supported in part through an NWO-VICI career grant (project number 639.043.308). The NRAO is a facility of the NSF operated under cooperative agreement by Associated Universities, Inc. Based on observations made with the NASA/ESA Hubble Space Telescope, obtained from the data archive at the Space Telescope Science Institute. STScI is operated by the Association of Universities for Research in Astronomy, Inc. under NASA contract NAS 5-26555. Some of the data presented herein were obtained at the W. M. Keck Observatory, which is operated as a scientific partnership among the California Institute of Technology, the University of California and the National Aeronautics and Space Administration. The Observatory was made possible by the generous financial support of the W. M. Keck Foundation. The authors wish to recognize and acknowledge the very significant cultural role and reverence that the summit of Mauna Kea has always had within the indigenous Hawaiian community. We are most fortunate to have the opportunity to conduct observations from this mountain.

\section{REFERENCES}

Auger M. W., Treu T., Brewer B. J., Marshall P. J., 2011, MNRAS, 411, L6 Biggs A. D., Wucknitz O., Porcas R. W., Browne I. W. A., Jackson N. J., Mao S., Wilkinson P. N., 2003, MNRAS, 338, 599

Bradač M., Schneider P., Steinmetz M., Lombardi M., King L. J., Porcas R., 2002, A\&A, 388, 373

Brewer B. J., et al., 2012, MNRAS, 422, 3574 




Figure 2. (a) $K^{\prime}$-band AO imaging of B1555+375 with contours from the MERLIN observations (red) of Marlow et al. (1999) and the VLBA observations presented here (blue) overlaid. (b) $K^{\prime}$-band AO image with the locations of the four radio components marked with circles. (c) Observed radio (blue pluses) and model-predicted (red open circles) image positions of B1555+375. The lens-plane critical curves are shown with the blue solid line and the source-plane caustics are shown with the red dotted line. The position of the source is at $(-0.1953,-0.1494)$, which is marked by the red filled circle. The centroid positions of the two mass components of the lens (a SIE and an exponential disc) are marked by the black filled triangles.

Table 2. A summary of the observational constraints and the predicted lens-model parameters. The lensed image positions in the radio (Marlow et al. 1999) (in arcsec) and average flux ratios (Koopmans et al. 2003) are with respect to image A. Uncertainties in the position measurements for images A, B and C are 0.001 arcsec and for image D are 0.006 arcsec.

\begin{tabular}{cccccccc}
\hline Image & \multicolumn{3}{c}{ Observed } & \multicolumn{4}{c}{ Model-Predicted } \\
& \multicolumn{3}{c}{ MERLIN 5 GHz } & & SIE+ExpDisc & SIE-only \\
& East & North & Flux ratio & East & North & Flux ratio & Flux ratio \\
\hline A & 0 & 0 & 1 & +0.000 & +0.000 & 1 & 1 \\
B & -0.0726 & +0.0480 & $0.620 \pm 0.039$ & -0.0715 & +0.0487 & 0.606 & 0.971 \\
C & -0.4117 & -0.0280 & $0.507 \pm 0.030$ & -0.4115 & -0.0278 & 0.445 & 0.312 \\
D & -0.1619 & -0.3680 & $0.086 \pm 0.024$ & -0.1693 & -0.3704 & 0.123 & 0.106 \\
\hline
\end{tabular}

Table 3. Best-fit model parameters and uncertainties for our two-component SIE + exponential disc lens model (our single SIE model is effectively equivalent to the model in Marlow et al. (1999) and so is not shown here). The positions are measured in arcsec, relative to the MERLIN radio position of image A. The ellipticity is defined as $e=1-q$ and the position angles are measured in degrees east of north. The Einstein radius $b$ and disc scale radius $\left(R_{s}\right)$ are measured in arcsec.

\begin{tabular}{ccc}
\hline Parameter & SIE Component & ExpDisc Component \\
\hline$\Delta$ RA & $-0.177 \pm 0.003$ & -0.162 (fixed $)$ \\
$\Delta$ Dec & $-0.205 \pm 0.004$ & -0.206 (fixed $)$ \\
$b$ & $0.178 \pm 0.004$ & $\ldots$ \\
$\kappa_{0}$ & $\ldots$ & $0.273 \pm 0.003$ \\
$e$ & $0.25 \pm 0.05$ & 0.83 (fixed) \\
$\theta$ & $4.9 \pm 1.3$ & 8.0 (fixed $)$ \\
$R_{s}$ & $\ldots$ & $0.236 \pm 0.003$ \\
\hline
\end{tabular}

Brewer B. J., Marshall P. J., Auger M. W., Treu T., Dutton A. A., Barnabè M., 2014, MNRAS, 437, 1950

Browne I. W. A., et al., 2003, MNRAS, 341, 13

Chen J., Rozo E., Dalal N., Taylor J. E., 2007, ApJ, 659, 52

Congdon A. B., Keeton C. R., 2005, MNRAS, 364, 1459

Dalal N., Kochanek C. S., 2002, ApJ, 572, 25

Evans N. W., Witt H. J., 2003, MNRAS, 345, 1351

Fukugita M., Futamase T., Kasai M., Turner E. L., 1992, ApJ, 393, 3

Jackson N., Bryan S. E., Mao S., Li C., 2010, MNRAS, 403, 826

Keeton C. R., 2001, ArXiv astro-ph/0102341,

Keeton C. R., Kochanek C. S., 1998, ApJ, 495, 157

Klypin A., Kravtsov A. V., Valenzuela O., Prada F., 1999, ApJ, 522, 82

Kochanek C. S., 1991, ApJ, 373, 354
Kochanek C. S., Dalal N., 2004, ApJ, 610, 69

Koopmans L. V. E., 2005, MNRAS, 363, 1136

Koopmans L. V. E., de Bruyn A. G., 2000, A\&A, 358, 793

Koopmans L. V. E., et al., 2003, ApJ, 595, 712

Koposov S. E., Belokurov V., Torrealba G., Wyn Evans N., 2015, preprint, (arXiv: 1503.02079)

Lagattuta D. J., Auger M. W., Fassnacht C. D., 2010, ApJ, 716, L185

Lagattuta D. J., Vegetti S., Fassnacht C. D., Auger M. W., Koopmans L. V. E., McKean J. P., 2012, MNRAS, 424, 2800

Maller A. H., Flores R. A., Primack J. R., 1997, ApJ, 486, 681

Mao S., Schneider P., 1998, MNRAS, 295, 587

Marlow D. R., et al., 1999, AJ, 118, 654

McKean J. P., et al., 2007, MNRAS, 378, 109

Metcalf R. B., Madau P., 2001, ApJ, 563, 9

Mittal R., Porcas R., Wucknitz O., 2007, A\&A, 465, 405

Möller O., Hewett P., Blain A. W., 2003, MNRAS, 345, 1

Moore B., Ghigna S., Governato F., Lake G., Quinn T., Stadel J., Tozzi P., 1999, ApJ, 524, L19

More A., McKean J. P., More S., Porcas R. W., Koopmans L. V. E., Garrett M. A., 2009, MNRAS, 394, 174

Myers S. T., et al., 2003, MNRAS, 341, 1

Nierenberg A. M., Treu T., Wright S. A., Fassnacht C. D., Auger M. W., 2014, MNRAS, 442, 2434

Papastergis E., Martin A. M., Giovanelli R., Haynes M. P., 2011, ApJ, 739,38

Ros E., Guirado J. C., Marcaide J. M., Pérez-Torres M. A., Falco E. E., Muñoz J. A., Alberdi A., Lara L., 2000, A\&A, 362, 845

Springel V., et al., 2008, MNRAS, 391, 1685

Strigari L. E., Bullock J. S., Kaplinghat M., Diemand J., Kuhlen M., Madau P., 2007, ApJ, 669, 676

Suyu S. H., et al., 2012, ApJ, 750, 10

The DES Collaboration et al., 2015, preprint, (arXiv: 1503.02584) 
Treu T., Dutton A. A., Auger M. W., Marshall P. J., Bolton A. S., Brewer B. J., Koo D. C., Koopmans L. V. E., 2011, MNRAS, 417, 1601

Turner E. L., Ostriker J. P., Gott III J. R., 1984, ApJ, 284, 1

Vegetti S., Koopmans L. V. E., 2009, MNRAS, 392, 945

Vegetti S., Lagattuta D. J., McKean J. P., Auger M. W., Fassnacht C. D., Koopmans L. V. E., 2012, Nature, 481, 341

Vegetti S., Koopmans L. V. E., Auger M. W., Treu T., Bolton A. S., 2014, MNRAS, 442, 2017

Winn J. N., Rusin D., Kochanek C. S., 2004, Nature, 427, 613

Xu D. D., Mao S., Cooper A. P., Gao L., Frenk C. S., Angulo R. E., Helly J., 2012, MNRAS, 421, 2553

Xu D., Sluse D., Gao L., Wang J., Frenk C., Mao S., Schneider P., Springel V., 2015, MNRAS, 447, 3189

This paper has been typeset from a $\mathrm{T}_{\mathrm{E}} \mathrm{X} / \mathrm{L} \mathrm{T}_{\mathrm{E}} \mathrm{X}$ file prepared by the author. 